\title{
Comparison of laryngeal mask airway and endotracheal intubation in gynecological cancer operation
}

\author{
ZHENGHUAN SONG ${ }^{1}$, JING TAN $^{1}$, JIA FANG $^{2}$, QINGMING BIAN $^{1}$ and LIANBING GU ${ }^{1}$ \\ ${ }^{1}$ Department of Anesthesia, Jiangsu Cancer Hospital; ${ }^{2}$ Department of Endocrinology, \\ Nanjing Traditional Chinese Medicine Hospital, Nanjing, Jiangsu 210009, P.R. China
}

Received May 4, 2018; Accepted November 27, 2018

DOI: $10.3892 / \mathrm{ol} .2018 .9813$

\begin{abstract}
Endotracheal intubation (ETI) and laryngeal mask airway (LMA) in terms of hemodynamics and reaction were compared. A total of 54 general anesthesia patients were randomized into two groups with 27 cases in each group. Acceleration index (ACI), cardiac index (CI), cardiac output (CO), left cardiac work (LCW), systemic circulation resistance (SVR), mean arterial pressure (MAP), heart rate (HR), systolic blood pressure (SBP) and diastolic blood pressure (DBP) were recorded at 12 time-points: before surgery (T0), start effect (T1), lost consciousness (T2), before ETI or LMA (T3), 1 min (T4), $3 \mathrm{~min}$ (T5), $5 \mathrm{~min}$ (T6) after ETI or LMA, the beginning of surgery (T7), 30 min (T8), 1 hour (T9) after surgery, the end of surgery (T10) and extubation (T11). In each group these indexes went down and rose up gently during surgery except for T4 (intubation) and T11 (extubation) in ETI. These indexes reached the highest at T11 (extubation). This is due to the stimulus on mucosa and muscle of root of tongue, throat and epiglottis from the windpipe. The stimulus excites sympathetic nerve and increases the release of catecholamine. As a result, the heart beats faster and blood pressure rises. However, the range in the LMA group is smaller especially at T4 and T11. This is most likely due to LMA not stimulating the trachea. SVR, MAP, HR, SBP and DBP were lower in LMA with statistical significance in some time-points. The other indexes such as ACI, CI, CO and LCW were significantly higher in LMA $(\mathrm{P}<0.05)$. These results indicated that LMA can be suitable for use in general anesthesia for less stimulation. The airway with LMA in patients undergoing gynecological cancer operation is better than ETI in keeping stable hemodynamics and producing less anesthetic complications with smooth recovery from general anesthesia.
\end{abstract}

Correspondence to: Dr Lianbing Gu, Department of Anesthesia, Jiangsu Cancer Hospital, 42 Baiziting, Xuanwu Section, Nanjing, Jiangsu 210009, P.R. China

E-mail: efjex964@163.com

Key words: laryngeal mask airway, endotracheal intubation, hemodynamics, gynecological cancer

\section{Introduction}

Elective lower abdominal surgery is frequently performed under general anesthesia among gynecological cancer patient population. Gynecology tumor patients are mostly middle-aged and old, and they often have internal diseases such as hypertension, cardiovascular and cerebrovascular disease and other medical problems. Anesthetization is highly demanding in these operations due to the long time, wide expansion and large wound. Thus, it is important to choose the appropriate airway management.

Developments in anesthesia practice and airway management have increased safety of pediatric anesthesia $(1,2)$. We improved airway management efficiency by using endotracheal intubation (ETI) or laryngeal mask airway (LMA) (3). There has been progress in the development of the design in the past ten years, changes in production material, and availability of various types. Anesthetists widely use ETI because of the advantages of good seal at the cricoid rings, decreased pressure, and thus a lower risk for mucosal injury (4). However, subglottic stenosis caused by a lack of cuff related mucosal perfusion due to cuff tube use, making their use controversial. In contrast, LMA has the advantages of a lack of direct contact with the trachea, no requirement for direct laryngoscopy, and a lower incidence of coughing (5-7).

We compared hemodynamics and respiratory function between LMA and ETI in gynecological cancer patients undergoing elective lower abdominal surgery $(8,9)$.

\section{Patients and methods}

Study design. A prospective, double-blind, randomized clinical trial was performed to evaluate the usefulness of the LMA compared to ETI by analyzing changes in clinical symptoms before and after surgery. The Ethics Committee of Jiangsu Cancer Hospital (Nanjing, China) approved the study.

Patients. After obtaining informed consents, 54 women, aged 32-55 years, with cervical and ovarian cancer were included in the study. The patients were scheduled for lower abdominal surgery at Jiangsu Cancer Hospital, between June 2012 and December 2012. The patient who took part in our research were randomized from 1 to 100 . Then they were divided into two groups according the odd/even 


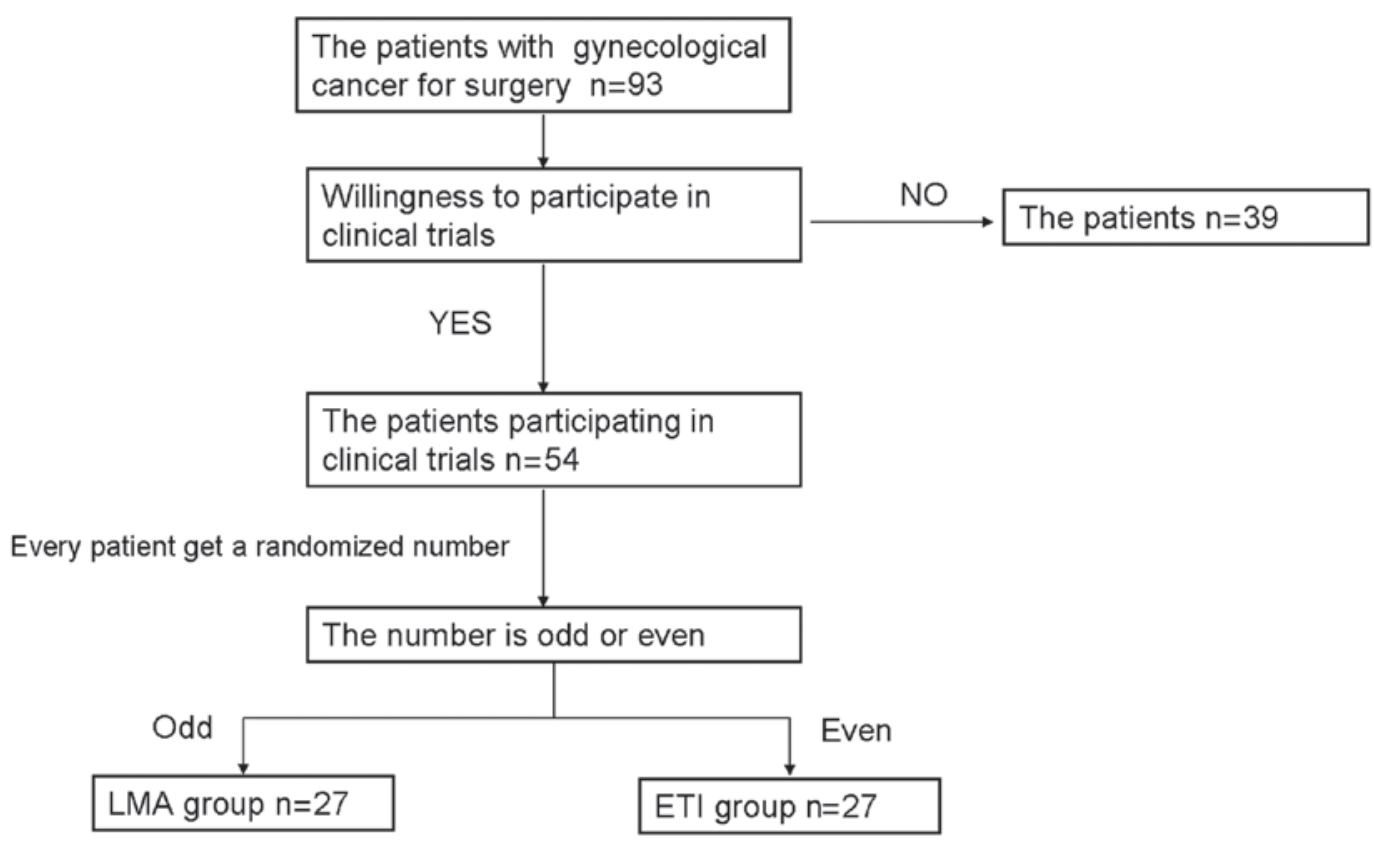

Figure 1. The diagram demonstrates the patient recruitment. ETI, endotracheal intubation; LMA, laryngeal mask airway.

numbers (Fig. 1). Then, the 54 patients were randomized into the LMA group $(n=27)$ and ETI group $(n=27)$. Patients with congenital abnormalities, risk of aspiration, upper respiratory tract infection, acute or chronic pulmonary diseases, or risk for difficult intubation were excluded from the study.

Statistical analysis. Continuous data were expressed as the number of participants $(\mathrm{n})$, mean $\pm \mathrm{SD}$, whereas categorical data were expressed as frequencies and percentages. Comparisons between the two groups were done using independent Student's t-test. Statistical analysis was carried out using Statistical Product and Service Solutions (SPSS) 16.0 (SPSS Inc., Chicago, IL, USA). A P-value $<0.05$ was considered statistically significant.

\section{Results}

A total of 54 patients were randomized in this study: 27 in the LMA group and 27 in the ETI group. The sex and age of the two groups were similar. The demographic and surgical data are presented in Table I.

Acceleration index (ACI), cardiac index (CI), cardiac output (CO), left cardiac work ( $\mathrm{LCW})$, systemic circulation resistance (SVR), mean arterial pressure (MAP), heart rate (HR), systolic blood pressure (SBP) and diastolic blood pressure (DBP) were recorded at 12 time-points: before surgery (T0), start effect (T1), lost consciousness (T2), before ETI or LMA (T3), 1 min (T4), 3 min (T5), 5 min (T6) after ETI or LMA, the beginning of surgery (T7), $30 \mathrm{~min}$ (T8), 2 hours (T9) after surgery, the end of surgery (T10) and extubation (T11) (Table II).

We found that in each group these indexes declined and then increased gently during surgery except for T4 and T12 especially in the ETI group. These indexes reached the highest at T11 (extubation). This is due to the stimulus on mucosa and muscle of root of tongue, throat and epiglottis from the windpipe. The stimulus excites sympathetic nerve and increases the release of catecholamine. As a result, the heart beats faster and blood pressure rises. However, the range in the LMA group is smaller especially at T4 and T11. This is most likely due to LMA not stimulating the trachea. ACI, CI, $\mathrm{CO}$ and LCW were higher in LMA with statistical significance at certain time-points $(\mathrm{P}<0.05)$ (Fig. 2A-D). Indexes such as SVR, MAP, HR, SBP and DBP were significantly higher in ETI (Fig. 2E-I).

\section{Discussion}

LMA was developed by A. Brain in 1981, and since then, it has flourished in practice and has been used to treat millions of patients worldwide $(10,11)$. The LMA provides more hands-free anesthesia than a facemask does, avoids many morbidities associated with tracheal intubation because there is no stress from the laryngoscope, and allows a faster recovery that does not require muscle relaxation $(12,13)$. The LMA has become an important choice for routine use, particularly in outpatient surgeries (14-16).

In our investigation, we assessed the eligibility of LMA by comparing hemodynamics and respiratory function between LMA and ETI in gynecological cancer operation. The results showed that LMA can be used safely and induces less stress reaction in gynecological cancer patients undergoing lower abdominal surgery. The correlated clinical indexes recorded in 12 time-points declined and then increased gently during surgery except for the time-point of intubation (T4) and extubation (T11) especially in the ETI group. These indexes reached the highest at extubation (T11). This is due to the stimulus on mucosa and muscle of root of tongue, throat and epiglottis, from the windpipe. The stimulus excites sympathetic nerve and increases the release of catecholamine (17-21). As a result, hearts beat faster and blood pressure rises. However, the range in the LMA group 
Table I. Demographic and surgical data.

\begin{tabular}{lcr}
\hline Items & LMA & ETI \\
\hline Age (year) & $44.30 \pm 8.05$ & $46.81 \pm 8.41$ \\
Sex (F/M, no.) & $28 / 0$ & $28 / 0$ \\
Weight (kg) & $60.22 \pm 7.73$ & $61.93 \pm 8.87$ \\
Height (cm) & $160.93 \pm 4.75$ & $159.19 \pm 3.94$ \\
Surgery time (min) & $125.19 \pm 17.70$ & $110.85 \pm 15.40$ \\
Total anesthesia time (min) & $137.56 \pm 17.92$ & $121.22 \pm 16.28$
\end{tabular}

LMA, laryngeal mask airway; ETI, endotracheal intubation.
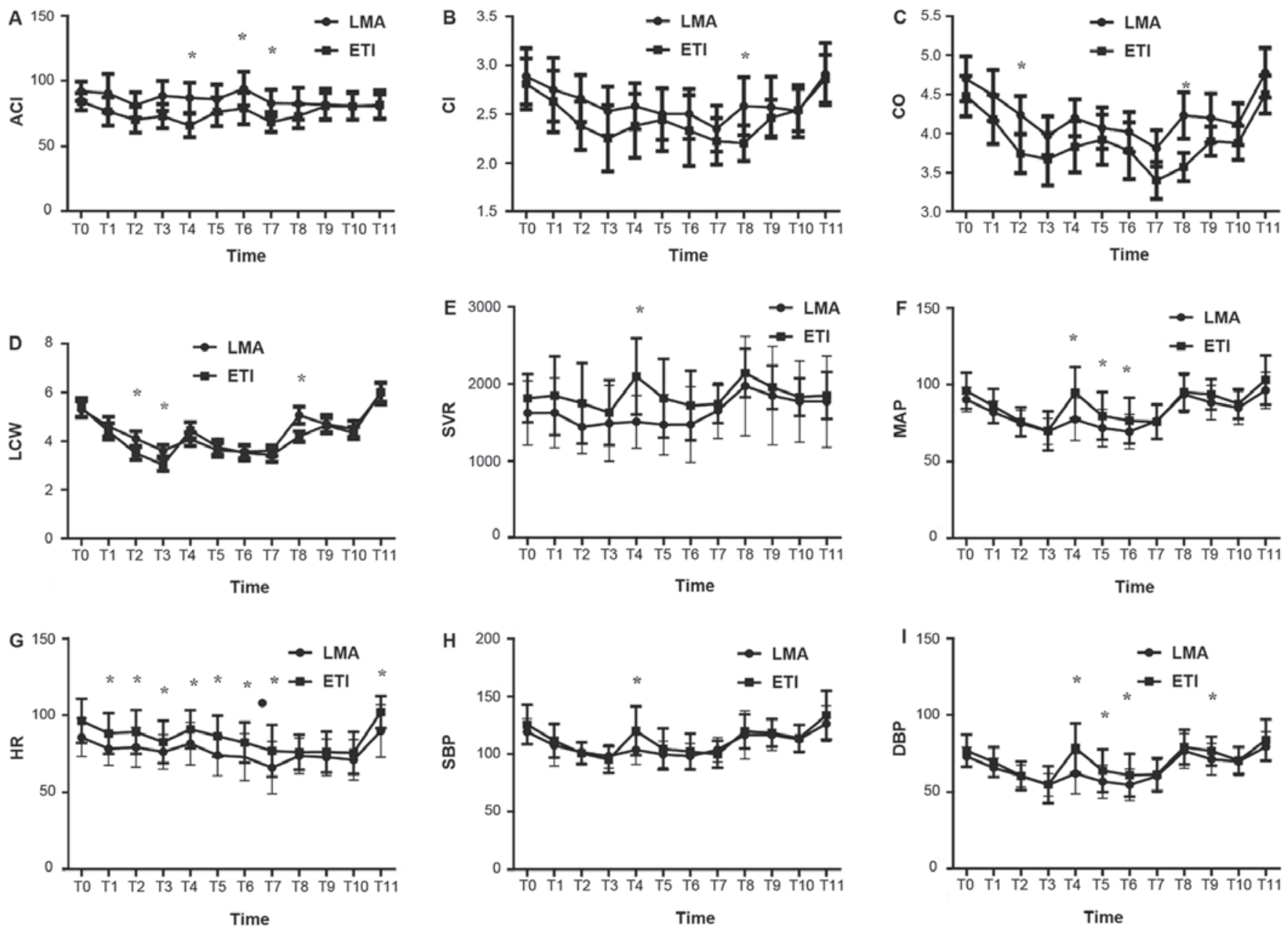

Figure 2. Comparison of clinical indexes between the two groups. (A) ACI of the 2 groups at each time-point as indicated; (B) CI of the 2 groups at the time-points; (C) $\mathrm{CO}$ of the 2 groups at the time-points; (D) LCW of the 2 groups at time-points; (E) SVR of the 2 groups at time-points; (F) MAP of the 2 groups at time-points; (G) HR of the 2 groups at time-points; (H) SBP of the 2 groups at time-points; (I) DBP of the 2 groups at time-points. "P<0.05; ACI, acceleration index; CI, cardiac index; CO, cardiac output; LCW, left cardiac work; SVR, systemic circulation resistance; MAP, mean arterial pressure; HR, heart rate; SBP, systolic blood pressure; DBP, diastolic blood pressure; ETI, endotracheal intubation; LMA, laryngeal mask airway.

is smaller especially at T4 and T11. This is possibly due to LMA not stimulating the trachea (22-25). One weakness of our design is that we could not get meaningful results using a multivariate logistic regression analysis. This may have been caused by the small number of samples. We will use a multivariate logistic regression analysis in future studies when a larger number of samples is available.
There were higher incidences of bucking in the ETI group in similar anesthesia in extubation. In addition, there were more postoperative complications in the ETI group such as pharyngalgia compared with LMA. This may be relevant to improper operation or excessive inflation of the catheter sleeve. This indicates that the stimulation of ETI is stronger than LMA. Most patients with ETI experience varying 


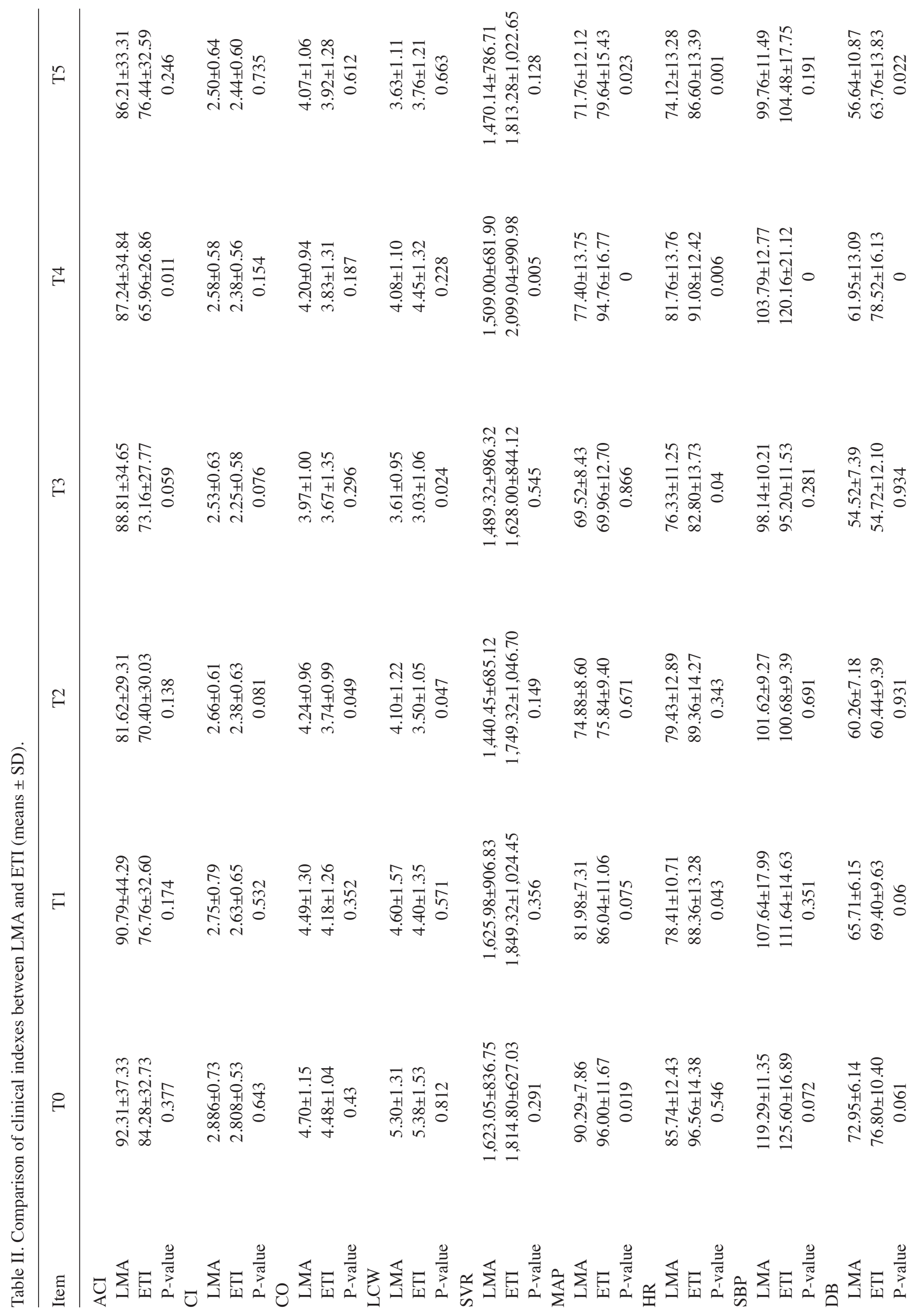




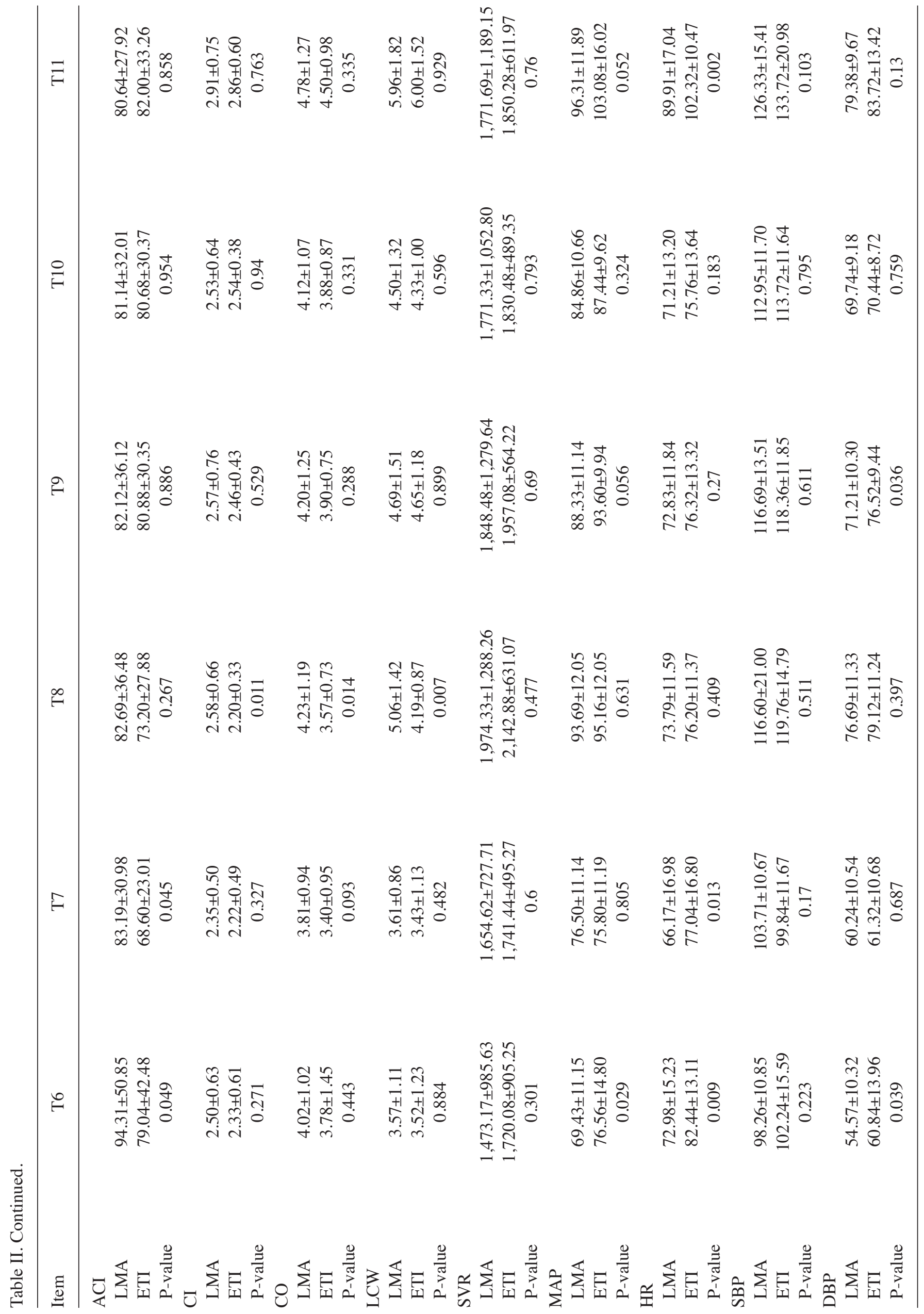


degrees of pharyngeal pain and have significant change in HR. However, patients with LMA do not have this complication. This is because LMA does not need to use laryngoscope and there is no need to enter trachea, thus avoid damaging tracheal mucosa and influencing circulation system. LMA has become complementary technology of ETI to offer a safe, effective, and simple ventilation method especially to the patient with microstomia. LMA is simpler to operate than ETI for medical workers and gains more time during emergency treatment. In addition, LMA is specifically suitable for the patient with microstomia (26).

In conclusion, the LMA may be a suitable method for airway management of patients with gynecological cancer under-going lower abdominal surgery $(27,28)$. Furthermore, LMA is a good alternative to ETI with easier insertion, and lower incidence of cardiovascular response.

\section{Acknowledgements}

Not applicable.

\section{Funding}

No funding was received.

\section{Availability of data and materials}

All data generated or analyzed during this study are included in this published article.

\section{Authors' contributions}

JT and LG designed the study and performed the experiments, ZS, JF and QB collected the data, JT and ZS analyzed the data, JT and LG prepared the manuscript. All authors read and approved the final manuscript.

\section{Ethics approval and consent to participate}

This study was approved by the Ethics Committee of Jiangsu Cancer Hospital (Nanjing, China). Signed informed consents were obtained from the patients or their guardians.

\section{Patient consent for publication}

Not applicable.

\section{Competing interests}

The authors declare that they have no competing interests.

\section{References}

1. Badr A, Tobias JD, Rasmussen GE, Stokes DC, Neblett WW III and Campbell P: Bronchoscopic airway evaluation facilitated by the laryngeal mask airway in pediatric patients. Pediatr Pulmonol 21: 57-61, 1996.

2. Khine HH, Corddry DH, Kettrick RG, Martin TM, McCloskey JJ, Rose JB, Theroux MC and Zagnoev M: Comparison of cuffed and uncuffed endotracheal tubes in young children during general anesthesia. Anesthesiology 86: 627-631, discussion 27A, 1997.
3. Junger A, Klasen J, Hartmann B, Benson M, Röhrig R, Kuhn D and Hempelmann G: Shorter discharge time after regional or intravenous anaesthesia in combination with laryngeal mask airway compared with balanced anaesthesia with endotracheal intubation. Eur J Anaesthesiol 19: 119-124, 2002.

4. Kihara S, Brimacombe J, Yaguchi Y, Watanabe S, Taguchi N and Komatsuzaki T: Hemodynamic responses among three tracheal intubation devices in normotensive and hypertensive patients. Anesth Analg 96: 890-895, 2003.

5. Pinosky M: Laryngeal mask airway: Uses in anesthesiology. South Med J 89: 551-555, 1996.

6. Landsman IS: The laryngeal mask airway. Int Anesthesiol Clin 35: 49-65, 1997.

7. Pennant JH and White PF: The laryngeal mask airway. Its uses in anesthesiology. Anesthesiology 79: 144-163, 1993.

8. Doksrød S, Løfgren B, Nordhammer A, Svendsen MV, Gisselsson L and Raeder J: Reinforced laryngeal mask airway compared with endotracheal tube for adenotonsillectomies. Eur J Anaesthesiol 27: 941-946, 2010.

9. Webster AC, Morley-Forster PK, Janzen V, Watson J, Dain SL, Taves D and Dantzer D: Anesthesia for intranasal surgery: A comparison between tracheal intubation and the flexible reinforced laryngeal mask airway. Anesth Analg 88: 421-425, 1999.

10. van Zundert TC, Brimacombe JR, Ferson DZ, Bacon DR and Wilkinson DJ: Archie Brain: Celebrating 30 years of development in laryngeal mask airways. Anaesthesia 67: 1375-1385, 2012.

11. Hernandez MR, Klock PA Jr and Ovassapian A: Evolution of the extraglottic airway: A review of its history, applications, and practical tips for success. Anesth Analg 114: 349-368, 2012.

12. Alexander CA: A modified intavent laryngeal mask for ENT and dental anaesthesia. Anaesthesia 45: 892-893, 1990.

13. Jakobsson J: The airway in day surgery. Minerva Anestesiol 76: 38-44, 2010.

14. Shavit I, Aviram E, Hoffmann Y, Biton O and Glassberg E: Laryngeal mask airway as a rescue device for failed endotracheal intubation during scene-to-hospital air transport of combat casualties. Eur J Emerg Med 25: 368-371, 2018.

15. Yang C, Zhu X, Lin W, Zhang Q, Su J, Lin B, Ye H and Yu R: Randomized, controlled trial comparing laryngeal mask versus endotracheal intubation during neonatal resuscitation - a secondary publication. BMC Pediatr 16: 17, 2016.

16. Benger JR, Voss S, Coates D, Greenwood R, Nolan J, Rawstorne S, Rhys $\mathrm{M}$ and Thomas M: Randomised comparison of the effectiveness of the laryngeal mask airway supreme, i-gel and current practice in the initial airway management of prehospital cardiac arrest (REVIVE-Airways): a feasibility study research protocol. BMJ Open 3: pii e002467, 2013.

17. Leventis C, Chalkias A, Sampanis MA, Foulidou X and Xanthos T: Emergency airway management by paramedics: Comparison between standard endotracheal intubation, laryngeal mask airway, and I-gel. Eur J Emerg Med 21: 371-373, 2014.

18. Xu R, Lian Y and Li WX: Airway complications during and after general anesthesia: a comparison, systematic review and Meta-Analysis of using flexible laryngeal mask airways and endotracheal tubes. PLoS One 11: e0158137, 2016.

19. Gasteiger L, Ofner S, Stögermüller B, Ziegler B, Brimacombe J and Keller C: Randomized crossover study assessing oropharyngeal leak pressure and fiber optic positioning: Laryngeal Mask Airway Supreme ${ }^{\mathrm{TM}}$ versus Laryngeal Tube LTS II ${ }^{\mathrm{TM}}$ size 2 in non-paralyzed anesthetized children. Anaesthesist 65 : 585-589, 2016.

20. Ozden ES, Meco BC, Alanoglu Z and Alkis N: Comparison of ProSeal laryngeal mask airway (PLMA) with cuffed and uncuffed endotracheal tubes in infants. Bosn J Basic Med Sci 16: 286-291, 2016.

21. Chun BJ, Bae JS, Lee SH, Joo J, Kim ES and Sun DI: A prospective randomized controlled trial of the laryngeal mask airway versus the endotracheal intubation in the thyroid surgery: evaluation of postoperative voice, and laryngopharyngeal symptom. World $\mathbf{J}$ Surg 39: 1713-1720, 2015.

22. Wheeler M: ProSeal laryngeal mask airway in 120 pediatric surgical patients: a prospective evaluation of characteristics and performance. Paediatr Anaesth 16: 297-301, 2006.

23. Sinha A, Sharma B and Sood J: ProSeal as an alternative to endotracheal intubation in pediatric laparoscopy. Paediatr Anaesth 17: 327-332, 2007. 
24. Goldmann K, Roettger C and Wulf H: Use of the ProSeal laryngeal mask airway for pressure-controlled ventilation with and without positive end-expiratory pressure in paediatric patients: a randomized, controlled study. $\mathrm{Br} \mathrm{J}$ Anaesth 95: 831-834, 2005

25. Hanna SF, Mikat-Stevens M, Loo J, Uppal R, Jellish WS and Adams M: Awake tracheal intubation in anticipated difficult airways: LMA Fastrach vs flexible bronchoscope: a pilot study. J Clin Anesth 37: 31-37, 2017.

26. van Esch BF, Stegeman I and Smit AL: Comparison of laryngeal mask airway vs tracheal intubation: A systematic review on airway complications. J Clin Anesth 36: 142-150, 2017.
27. Al-Mazrou KA, Abdullah KM, ElGammal MS, Ansari RA, Turkistani A and Abdelmeguid ME: Laryngeal mask airway vs. uncuffed endotracheal tube for nasal and paranasal sinus surgery: Paediatric airway protection. Eur J Anaesthesiol 27: 16-19, 2010. 28. Ong M, Chambers NA, Hullet B, Erb TO and von UngernSternberg BS: Laryngeal mask airway and tracheal tube cuff pressures in children: Are clinical endpoints valuable for guiding inflation? Anaesthesia 63: 738-744, 2008.

(i) () $९$ This work is licensed under a Creative Commons cc) ${ }_{\mathrm{EY} \text { NO ND }}$ Attribution-NonCommercial-NoDerivatives 4.0 International (CC BY-NC-ND 4.0) License. 